\title{
Research on Self-Regulated Learning by Using Network Learning Space for Cross-Disciplinary MPAcc Postgraduate Freshmen Based on HeXie Management Theory
}

\author{
Xiaozhuo Wei ${ }^{1, *}$ Yunpeng $\mathrm{Li}^{1}$, Wenjun Shao ${ }^{1}$, Xing Dong ${ }^{1}$ \\ ${ }^{1}$ School of Economics and Management, Jiangsu University of Science and Technology, Zhenjiang, Jiangsu 212003, \\ China \\ *Corresponding author. Email: wwwxxx0915@sina.com
}

\begin{abstract}
The cross-disciplinary postgraduates are a special group with a large proportion for MPAcc. Compared with students of accounting background, their accounting knowledge and skills at the time of admission are far from satisfactory. The use of network learning space by cross-disciplinary MPAcc postgraduate freshmen for self-learning and self-improvement is an effective way to address the difficulties. Based on HeXie management theory, this paper pragmatically explores the above problems and puts forward corresponding suggestions.
\end{abstract}

Keywords: cross-disciplinary MPAcc postgraduate freshman, network learning space, self-regulated learning, HeXie management theory

\section{和谐管理视角下跨专业MPAcc研究生新生利用网络学习 空间自主学习探究}

\author{
魏晓卓 ${ }^{1, *}$ 李云鹏 $^{1}$ 郡文君 $^{1}$ 董星 $^{1}$
}

1江苏科技大学经济管理学院, 镇江, 江苏, 212003, 中国

“通讯作者Email: www.xxx0915@sina.com

中文摘要

跨专业研究生是MPAcc研究生中数量占比不容小觑的一个特殊群体, 其入学时的会计专业知识与技能 同会计学专业背景学生相比差距较大, 跨专业MPAcc研究生新生利用网络学习空间通过自主学习查漏 补缺与自我提升, 是解决其培养困境的一个有效路径。本研究基于和谐管理理论, 对上述问题进行了 实践探索，提出了相应的意见与建议。

关键词：跨专业MPACC研究生新生；网络学习空间；自主学习；和谐管理理论

\section{1. 跨专业MPAcc研究生新生培养的现实困境}

\section{1. 跨专业MPAcc研究生生源数量提升明显}

近年来, 报考会计硕士专业学位 (也称专业会计 硕士, Master of Professional Accounting, 简称 MPAcc）的学生数量大幅提升, 由于MPAcc研究生全 国初试时只考“英语”和“管理类联考综合能力”不考会
计学的相关内容、应届本科毕业生报考不受限制等原 因, 因此最终录取时跨专业生源占有很大比例（本研 究所界定的跨专业MPAcc研究生新生, 主要指本科所 学专业不是会计、财务管理、审计等与会计相关专业 的MPAcc研究生一年级学生)。

比如，以江苏 $\mathrm{J}$ 高校为例，在MPAcc 项目 $\mathrm{J}$ 高校首 年正式招生时, 跨专业生源就达到 $50 \%$, 研究生收费 改革的 2014 年这一比例也接近 $50 \%$, 其他年份的比例 一般为三分之一。 


\section{2. 基于和谐管理理论研究MPAcc 研究生培 养问题的优越性}

\section{2. 跨专业MPAcc 研究生新生培养存在诸多 困难}

对于跨专业MPAcc研究生新生来说, 一般情况下他 们只是在复试前突击自学了一些会计学初级的专业内 容, 虽然他们同会计学专业背景学生一样都参加了 “会计专业综合” 之类的复试考试, 但是其在会计知 识储备与技能积累的系统性、深入性等方面均存在较 大差距。

尽管各培养单位也采取了诸如要求跨专业MPAcc研 究生新生补修本科会计学课程、导师推荐经典书目让 新生进行自学等措施试图夯实其专业基础, 但由于培 养单位一般并不针对跨专业MPAcc 研究生设置特殊的培 养模式、研究生一年级自身课程时间与旁听校本部本 科课程时间冲突、需补修的本科课程可能不在研究生 所在校区、研究生个别课程因新生所选修人数太少无 法开设、看书自学对新生本身能力要求更高等多种原 因, 跨专业MPAcc 研究生的专业知识结构、能力、视野 以及培养效果与质量均存在较多的问题, 解决跨专业 MPAcc 研究生新生培养的现实困境就成为一项重要而急 迫的课题。

\section{2. 突破跨专业MPAcc研究生新生培养瓶颈的 和谐管理路径思考}

\section{1. 跨专业MPAcc 研究生新生利用网络学习 空间自主学习的可行性}

当前, 推进教育创新与变革的探索如火如茶, 基 于网络的教育教学创新越来越受到国内外的广泛重 视。《国家中长期教育改革和发展规划纲要（20102020年) 》提出 “开发网络学习课程, 创新网络教学 模式, 鼓励学生利用信息手段主动学习、自主学习, 增强运用信息技术分析解决问题能力” ${ }^{[1]}$ 的要求。

《教育部关于加强网络学习空间建设与应用的指导意 见》（教技（2018）16号）指出, 要引导学生 “根据 自身学习需要, 通过空间 (注: 即网络学习空间) 选 择网络课程、在线测试、智力资源服务等进行自主学 习，强化应用空间解决问题的意识” [2]。

由于网络学习空间融资源、服务与数据为一体 [3], 突破了大学校园的时空藩篱, 方便与促进研究生 进行弹性自主学习与高质量学习, 激发了研究生的学 习积极性, 有利于其专业知识消化吸收, 更有利于培 养其勤学不辍及勇于探索研究的精神。因此, 跨专业 MPAcc研究生作为信息素养较强的学习主体, 利用网 络学习空间进行自主学习, 不失为解决跨专业MPAcc 研究生新生培养瓶颈的有效路径。
事实上，随着互联网和人工智能等新技术的不断 发展, 面对高等教育环境日益增长的不确定性、模糊 性、复杂性和快变性，用传统管理理论的线性思维处 理方式解决高等教育管理问题越来越显得 “黔驴技 穷”，而中国学者席西民教授创立的和谐管理理论充 分展现了灵活性的特征, 该理论提出 ${ }^{[4]}$ : 要结合环境 变化、未来趋势与组织情境等确立 “愿景和使命”, 对于特定情境中的问题，应在遵从 “愿景和使命” 的 基础上根据当下的情境辨识 “和谐主题” ，并根据

“和谐主题” 来构建适当的 “和则”与 “谐则” 体系 以及 “耦合” 方式, 并在发展中根据环境和运行情况 不断进行动态调整。

在应用实践层面，席教授及其研究团队的和谐管 理理论研究成果更多地应用于企业当中, 对学校等其 他组织问题也有涉及, 例如文献[5]研究了博士生导师 对其博士生进行激励的相关问题，这对本研究基于和 谐管理理论开展跨专业MPAcc研究生新生利用网络学 习空间自主学习的应用分析给予了重要启示。

\section{3. 和谐管理视角下跨专业MPAcc研究生新生 利用网络学习空间自主学习的实践与建议}

\section{1. 愿景和使命}

“愿景和使命” 是组织发展的定位和长远目标, 一般具有相对稳定性和战略意义 ${ }^{[4,6-7]}$ 。

以 $\mathrm{J}$ 高校为例, 其MPAcc 项目的愿景是 “成为工科 院校中一流的MPAcc会计教育中心”，培养 “具有管理 洞察、国际视野、信息整合的高层次、高素质、应用 型高级会计人才”。这说明, 对会计学专业背景的 MPAcc 研究生与非会计学专业背景的跨专业MPAcc 研究 生来说, 最终的培养目标是相同的, 都是要 “培育职 业素养，造就业界精英”。

\section{2. 和谐主题}

“和谐主题” 是指组织在一定时期内最为迫切需 要完成的核心任务, 亦即工作重心或中心工作议题 ${ }^{[6-}$ ${ }^{8]}$ 。

对于如何培养新时代跨专业MPAcc 研究生, 通过对 $\mathrm{J}$ 高校研究生院院长、经济管理学院院长、MPAcc教育 中心主任、会计学专业负责人、财务管理专业负责人 等的访谈, 确定本研究的 “和谐主题” 是：应对新时 代和信息社会对MPAcc 研究生培养的迫切需求, MPAcc 研究生导师要指导跨专业新生充分利用网络学习空间 强化自主学习, 根据自身情况查漏补缺与自我提升, 构建合理的专业知识结构, 并熟练运用专业知识解决 
其经济管理学院应资助教师加快建设特色鲜明、质量 优良的会计类线上一流课程，并对一流课程负责人在 岗位津贴、职级和专业技术职务晋升等方面予以倾 斜; 要鼓励MPAcc研究生导师充分利用优质在线资 源, 对接国内知名在线开放课程平台, 改进教学方 法, 推进教学改革。研究生院和经济管理学院可在修 订甚至重构MPAcc研究生培养方案时, 增添与完善跨 专业MPAcc 研究生新生利用网络学习空间自主学习的 内容, 并在新增导师岗前培训、年度导师资格遴选工 作中对导师也提出相应要求。

\section{5. 和谐耦合}

“和谐耦合” 是在特定的 “和谐主题”下 “和 则” 与 “谐则” 在依赖、调节和转化等互动中不间断 地螺旋式推进的过程 ${ }^{[6-9]}$ 。

基于和谐管理思想 ${ }^{[6-9]}$, 就跨专业MPAcc研究生新 生利用网络学习空间自主学习中 “和则”与 “谐则” 的相互转化而言，示例如下:

\subsection{1. “谐则” 转 “和则” 示例}

随着 $\mathbf{J}$ 高校MPAcc 研究生培养方案的修订或重构以 及新增导师岗前培训制度、年度导师资格遴选制度等 的进一步完善, 利用网络学习空间加强学习的优越 性、如何利用网络学习空间的核心思想与内容等会逐 渐被MPAcc 研究生导师、跨专业MPAcc 研究生了解并 认同, 导师的工作积极性、研究生自主学习的主观能 动性得以提升, 进而变为其习惯, 这样, 相关制度中 的硬性规定转变为了软要素, 即 “谐则” 转变为 “和 则”

\subsection{2. “和则” 转 “谐则” 示例}

事实上，师生能动性、积极性的发挥，也可以发 现和弥补网络学习空间相关制度的不足与漏洞, J高校 及其研究生院、经济管理学院会进一步对相关制度进 行优化, 即对 “和则” 的深化部分在一定条件下又转 化为新的 “谐则” , 相关制度得以持续的完善与进 步。

\section{4. 结束语}

跨专业研究生是MPAcc研究生中需要重视的一个 特殊群体, 培养单位和导师可以运用和谐管理理论的 思路、工具与技术, 对跨专业MPAcc研究生培养进行 顶层设计, 科学合理地引导跨专业MPAcc 研究生新生 利用网络学习空间, 通过自主学习对其专业知识补短 板、强弱项, 进而将其培养成为高层次、高素质、应 用型高级会计人才。 
本研究将和谐管理理论从企业的适用范围进一步 扩展到高校研究生教育等管理领域, 研究跨专业 MPAcc 研究生新生利用网络学习空间自主学习的管理 问题，丰富了和谐管理的案例资源。

\section{致谢}

本文得到江苏省研究生教育教学改革课题 （JGZZ19_080）、江苏省高等教育学会应用型高校研 究专项课题 (2019JSZX005) 、江苏省高等教育教改 研究立项课题 (2019JSJG234) 、江苏省高校教学管理 研究课题 (JY201831) 的资助。

\section{REFERENCES}

[1] Office of the Working Group on the Outline of the National Medium-and Long-Term Education Reform and Development Plan, Outline of National Mediumand Long-Term Education Reform and Development Plan

(2010-2020), http://old.moe.gov.cn/publicfiles/business/htmlfiles/mo e/info_list/201407/xxgk_171904.html?authkey=gwbux, 2010-7-29.

[2] Ministry of Education of the People's Republic of China, Suggestions for the Ministry of Education on strengthening the construction and application of network learning space, http://www.moe.gov.cn/srcsite/A16/s3342/201901/t201 90124_367996.html, 2019-1-16.

[3] Ministry of Education of the People's Republic of China, Notice of the Ministry of Education on issuing Guide to the Construction and Application of Online Learning Space, http://www.moe.gov.cn/srcsite/A16/s3342/201805/t201 80502_334758.html, 2018-4-17.

[4] Y-M. Xi, P. Liu, The possibility and pathway of breakthrough for management in China: Exploration and engagement based on HeXie management research, Journal of Management Sciences in China, 22(9) (2019) $1-11$.

[5] Y-F. Shang, Y-M. Xi, J. Ma, L. Li, The research of leader's motivation on employee: Based on the harmonious sense-giving system-With outstanding doctoral tutor guiding graduates as an example, An Academic Edition of ManaMaga, (4) (2013) 3-17.

[6] Y-M. Xi, W. Han, J. Ge, et al., Research on HeXie Management Theory, Xi'an: Xi'an Jiaotong University Press, 2006.
[7] Y-M. Xi, J. Ge, et al., HeXie Management Theory: Cases and Implications, Xi'an: Xi'an Jiaotong University Press, 2006.

[8] X-Z. Wei, Research on Grain Financial Direct Subsidy Based on HeXie Management Theory, Ph.D. Thesis, Zhenjiang: Jiangsu University, 2015.

[9] X-J. Zhang, Y-M. Xi, J-L. Mao, Equipment procurement quality management on the basis of HeXie management theory, Journal of Industrial Engineering and Engineering Management, 26(2) (2012) 48-57. 